\title{
Reduction of porcine circovirus type 2 (PCV2) viremia by a reformulated inactivated chimeric PCV1-2 vaccine-induced humoral and cellular immunity after experimental PCV2 challenge
}

\author{
Hwi Won Seo, Yeonsu Oh, Kiwon Han, Changhoon Park and Chanhee Chae
}

\begin{abstract}
Background: The objective of the present study was to elucidate the humoral and cellular immune response mechanisms by which a reformulated inactivated chimeric PCV1-2 vaccine reduces the PCV2 viremia. Forty PCV2 seronegative 3-week-old pigs were randomly divided into the following four groups: vaccinated challenged (T01), vaccinated non-challenged (T02), non-vaccinated challenged (T03), and non-vaccinated non-challenged (T04) animals. The pigs in groups T01 and T02 were immunized with a reformulated inactivated chimeric PCV1-2 vaccine (Fostera $^{\text {TM }}$ PCV; Pfizer Animal Health) administered as a $2.0 \mathrm{ml}$ dose at 21 days of age. At 35 days of age (0 days post-challenge), the pigs in groups T01 and T03 were inoculated intranasally with $2 \mathrm{ml}$ each of PCV2b.
\end{abstract}

Results: A reduction of PCV2 viremia coincided with the appearance of both PCV2-specific neutralizing antibodies (NA) and interferon- $\gamma$-secreting cells (IFN- $\gamma-S C s$ ) in the vaccinated animals. However, the presence of anti-PCV2 IgG antibodies did not correlate with the reduction of PCV2 viremia. Lymphocyte subset analysis indicated that the numbers of $\mathrm{CD}^{+}$and $\mathrm{CD}^{+}$cells increased in vaccinated animals but the numbers of $\mathrm{CD} 4^{+}$cells decreased transiently in non-vaccinated animals. The observation of a delayed type hypersensitivity response in only the vaccinated animals also supports a $\mathrm{CD} 4^{+}$cell-associated protective cellular immune response induced by the reformulated inactivated chimeric PCV1-2 vaccine.

Conclusions: The induction of PCV2-specific NA and IFN- - -SCS, and CD4 ${ }^{+}$cells by the reformulated inactivated chimeric PCV1-2 vaccine is the important protective immune response leading to reduction of the PCV2 viremia and control of the PCV2 infection. To our knowledge this is the first demonstration of protective humoral and cellular immunity induced by the reformulated inactivated chimeric PCV1-2 vaccine and its effect on reduction of PCV2 viremia by vaccination.

Keywords: Chimeric PCV1-2 vaccine, Efficacy, Humoral and cellular immunity, Porcine circovirus-associated disease, Porcine circovirus type 2, Porcine circovirus vaccine

\footnotetext{
* Correspondence: swine@snu.ac.kr

College of Veterinary Medicine, Department of Veterinary Pathology, Seoul National University, 599 Gwanak-ro, Gwanak-gu, Seoul 151-742, Republic of Korea
} 


\section{Background}

Porcine circovirus type 2 (PCV2) is one of the most economically important swine pathogens worldwide. The first commercial PCV2 vaccine was used under special license in France and Germany in 2004, 13 years after postweaning multisystemic wasting syndrome (PMWS) was first identified and reported in western Canada [1]. In addition to PMWS, PCV2 is also associated with a number of diseases and syndromes, collectively referred to as porcine circovirusassociated disease (PCVAD) [2,3].

Currently, 5 commercial PCV2 vaccines are available worldwide and differ in their antigen [1]. One vaccine (Circovac, Merial) is based on the classical approach of an inactivated oil-adjuvanted vaccine. Three subunit vaccines (Circoflex, Boehringer Ingelheim; Circumvent PCV, Intervet/Merck; Porcillis PCV, Schering-Plough/ Merck) are based on an open reading frame 2 (ORF2; capsid) protein expressed in the baculovirus system. Another vaccine (Suvaxyn PCV2 One Dose, Pfizer Animal Health/Fort Dodge Animal Health) is based on a chimeric PCV1-2 virus containing the genomic backbone of the non-pathogenic PCV1, with the ORF2 capsid gene replaced by that of PCV2 [4]. In 2008, Pfizer Animal Health temporarily removed the inactivated chimeric PCV1-2 vaccine product from the markets because a chimeric PCV1-2 virus was incidentally detected in the field due to incomplete inactivation of the vaccine [5]. In August 2011, a reformulated version of the chimeric PCV1-2 vaccine under a new brand name (Fostera $^{\text {TM }}$ PCV, Pfizer Animal Health) re-entered the market.

PCV2 viremia plays a central role in the development of PMWS. High levels of PCV2 viremia are associated with the development of PCVAD [6,7]. Reductions in the PCV2 viremia have coincided with the appearance of both PCV2-specific neutralizing antibodies (NA) and interferon $\gamma$-secreting cells (IFN$\gamma$-SCs) in PCV2-infected animals [6-9]. Therefore, the induction of PCV2-specific NA and IFN- $\gamma-\mathrm{SCs}$ by commercial PCV2 vaccines is a critical parameter to evaluate the efficacy of PCV2 vaccines in the control of PCV2 infection. It has been reported that subunit and inactivated PCV2 vaccines elicit PCV2-specific NA and IFN- $\gamma$-SCs [10,11]. Although the former inactivated chimeric PCV1-2 vaccine induced PCV2specific NA [12], little is known regarding the protective immunity by which the reformulated inactivated chimeric PCV1-2 vaccine reduces the PCV2 viremia. The objective of present study is to elucidate the mechanisms how humoral and cellular immune response induced by the reformulated inactivated chimeric PCV1-2 vaccine reduces PCV2 viremia under experimental conditions.

\section{Methods}

\section{Animals and housing}

A total of 40 colostrum-fed, cross-bred, conventional piglets were purchased at 14 days of age from a commercial farm. All piglets were negative for porcine reproductive and respiratory syndrome virus (PRRSV) and Mycoplasma hyopneumoniae according to routine serological testing. All piglets were negative for PCV1-2a and PCV2 viremia by real-time polymerase chain reaction (PCR), respectively $[13,14]$. All piglets were also seronegative against PCV2 according to commercial ELISA (Synbiotics, Lyon, France). All pigs were housed in an environmentally controlled building as previously described [15].

\section{Experimental design}

A total of 40 piglets were randomly divided into 4 groups (10 pigs per group). The pigs in groups T01 and T02 were immunized with an inactivated chimeric PCV1-2 vaccine (Fostera ${ }^{\mathrm{TM}}$ PCV; Pfizer Animal Health Inc.) administered as a $2.0 \mathrm{ml}$ dose at 21 days of age based on the manufacturer's recommendations. At 35 days of age [0 days post-challenge $(\mathrm{dpc})$ ], the pigs in groups T01 and T03 were inoculated intranasally with $2 \mathrm{ml}$ each of a PCV2b [strain SNUVR000463; 5th passage; $1.0 \times 10^{5}$ tissue culture infective dose of $50 \%$ (TCID50)/ml]. The pigs in group T04 remained unvaccinated and unchallenged, and served as the negative control group. The pigs in each group were housed separately within the facility. Blood samples and nasal swabs were collected at $-14,0$, 14 , and $28 \mathrm{dpc}$. All pigs were euthanized for necropsy at $28 \mathrm{dpc}$ and superficial inguinal lymph nodes were collected for histopathology and immunohistochemistry. All of the methods were previously approved by the Seoul National University Institutional Animal Care and Use Committee.

\section{Quantification of PCV2 DNA in blood and nasal swab}

DNA was extracted from serum and nasal samples using the QIAamp DNA mini kit. DNA extracts were used to quantify PCV2 genomic DNA copy numbers by realtime PCR as previously described by Gagnon et al. (2008) [14]. DNA extracts from serum samples were also used to detect PCV1-2a DNA by real-time PCR as previously described by Shen et al. (2010) [13].

\section{Serology}

The serum samples were tested using the commercial PCV2 ELISA IgG (Synbiotics, Lyon, France) and serum virus neutralization (SVN) test [16].

\section{Preparation of PCV2 antigen}

The same PCV2 strain used for challenge in the present study, was propagated in PCV-free PK15 cells to a titer of $10^{4} \mathrm{TCID} 50 / \mathrm{ml}$ and treated with two freeze-thaw cycles. 
The PCV2 antigen was prepared by concentrating the virus present in cell culture by ultracentrifugation at $100,000 \mathrm{x} g$ at $4^{\circ} \mathrm{C}$ for $3 \mathrm{~h}$. The virus pellet was resuspended with PBS. The concentrated PCV2 was inactivated by exposing to an $8 \mathrm{~W}$ germicidal UV lamp at a distance of $15 \mathrm{~cm}$ for $1 \mathrm{~h}$. Immunoperoxidase assay was performed to confirm the inactivation of virus as previously described by Rodriguez-Arrioja et al. (2000) [17].

\section{Delayed type hypersensitivity}

The delayed type hypersensitivity (DTH) test was performed on 40 piglets from 4 groups at 5 weeks of age ( 2 weeks after vaccination; $0 \mathrm{dpc}$ ). Piglets were injected intradermally on the left inguinal area with $250 \mu \mathrm{g}$ of PCV2 antigen from infected PK15 cells. Phytohemaglutin (PHA; Roche Diagnostics $\mathrm{GmbH} ; 20 \mu \mathrm{g} / \mathrm{ml}$ in $0.1 \mathrm{ml}$ ) and saline $(0.1 \mathrm{ml})$ were used as positive and negative controls, respectively. The mean diameters of the induration at the test site were measured with a micrometer $36 \mathrm{~h}$ after injection.

\section{Direct immunofluorescence assay}

Cryosections (about $7 \mu \mathrm{m}$ in thickness) were prepared from skin biopsy specimens in DTH test. Sections were transferred to microscope slides treated with poly-L-lysine. The slides were fixed with acetone for $20 \mathrm{~min}$ and then air-dried. The slides were then covered with $50 \mu \mathrm{l}$ of mouse anti-pig CD4a conjugated with R-phycoerythrin (R-PE) (1:100 dilution; SouthernBiotech, Birmingham, AL, USA) and incubated for $60 \mathrm{~min}$ at $37^{\circ} \mathrm{C}$ in a moisture chamber. After three washing steps with PBS, the slides were mounted in buffered glycerin for observation by fluorescence microscopy.

\section{Enzyme-linked immunospot (ELISPOT) assay}

The numbers of PCV2-specific interferon- $\gamma$-secreting cells (IFN- $\gamma$-SCs) were determined in peripheral blood mononuclear cells (PBMCs) at $-14,0,14$, and $28 \mathrm{dpc}$ as previously described [18]. Briefly, $100 \mu \mathrm{l}$ containing $2 \times 10^{6}$ PBMCs in RPMI 1640 medium supplemented with $10 \%$ fetal bovine serum (HyClone Laboratories, Inc., SelectScience, Bath, UK) were seeded into plates pre-coated with anti-porcine IFN- $\gamma$ monoclonal antibody (5 $\mu \mathrm{g} / \mathrm{ml}, \mathrm{MABTECH}$, Mariemont, OH, USA) and incubated with $100 \mu \mathrm{l}$ of PCV2 antigen $(20 \mu \mathrm{g} / \mathrm{ml})$, phytohemagglutinin $(10 \mu \mathrm{g} / \mathrm{ml}$, Roche Diagnostics $\mathrm{GmbH}$, Mannheim, Germany) as a positive control, or PBS as a negative control for $40 \mathrm{~h}$ at $37^{\circ} \mathrm{C}$ in a $5 \%$ humidified $\mathrm{CO}_{2}$ atmosphere. Then, the wells were washed five times with PBS (200 $\mu \mathrm{l}$ per well). Thereafter, procedure was followed by manufacturer's instructions using a commercial ELISPOT assay kit (MABTECH, Mariemont, OH, USA). Spots on the membranes were read by an automated ELISpot reader (AID ELISpot Reader, AID
GmbH, Strassberg, Germany). The results were expressed as the number of responding cells per million PBMCs.

\section{Flow cytometry}

PBMCs were incubated with R-PE- or FITC-conjugated mouse monoclonal antibodies (antiswine CD3 [R-PE] and CD4 [R-PE and FITC]; SouthernBiotech, Birmingham, AL, USA) for $30 \mathrm{~min}$ at $4^{\circ} \mathrm{C}$ in the dark and washed twice with PBS containing $0.1 \%$ sodium azide and $0.1 \%$ bovine serum albumin. Cells stained with conjugated antibodies were resuspended immediately in supplemented RPMI 1640 medium. Cells were analyzed using a FACSCalibur flow cytometer (Becton Dickinson) as previously described by Sosa et al. (2009) [19].

\section{Histopathology and immunohistochemistry}

For the morphometric analysis of histopathological lesion score and PCV2 antigen score in superficial inguinal lymph nodes were collected at necropsy. In each sample, three sections were randomly selected and examined "blindly" as previously described by Kim et al. (2011) [15]. The scores of lesions in lymph nodes ranged from 0 (No lymphoid depletion or granulomatous replacement) to 5 (severe lymphoid depletion and granulomatous replacement), and the PCV2 antigen scores were obtained by counting the number of PCV2 positive cells per unit area $\left(0.25 \mathrm{~mm}^{2}\right)$.

\section{Statistical analysis}

Continuous data (DTH response, PCV2 DNA, PCV2 serology, PCV2-specific IFN- $\gamma$-SCs, and lymphocyte subsets) were analyzed with a one-way analysis of variance (ANOVA). If a one-way ANOVA was significant $(P<0.05)$, pairwise testing using Tukey's adjustment was performed. Discrete data (lymphoid lesion score and PCV2 antigen score, and proportion of viremic pigs) were analyzed by Chi-square and Fisher's exact test.

The Pearson's correlation coefficient was used to assess the relationship among viremia, serum virus neutralization titer, PCV2-specific IFN- $\gamma$-SCs, and the Spearman's rank correlation coefficient was used to assess lymphoid lesion score and PCV2 antigen score. A value of $p<0.05$ was considered significant.

\section{Results}

PCV2 DNA in sera and nasal swabs

PCV2 DNA was not detected in serum and nasal samples from any of pigs at $0 \mathrm{dpc}$. Vaccinated challenged (T01) animals had a significantly lower number of genomic copies of PCV2 in the blood than non-vaccinated challenged (T03) animals at 14 and 28 days post challenge (dpc; $p<0.001$, Figure 1). The percentage of viremic pigs was significantly lower in vaccinated challenged animals 


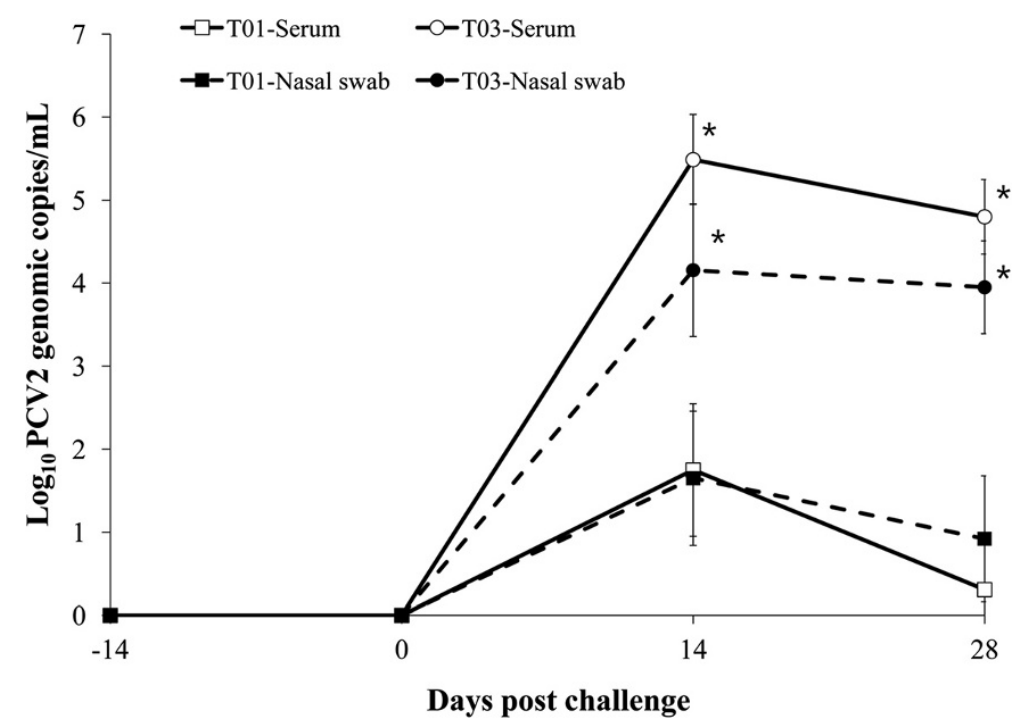

Figure 1 Mean values of the genomic copy number of porcine circovirus type 2 DNA in serum and nasal swabs from vaccinated challenged (T01; $\square$ for serum and $₫$ for nasal swab) and non-vaccinated challenged (T03; $\bigcirc$ for serum and $\bullet$ for nasal swab) animals. Variation is expressed as the standard deviation. Significant difference is indicated at $p$ value $<0.001^{*}$.

(4/10 at $14 \mathrm{dpc}$ and $2 / 10$ at $28 \mathrm{dpc}$ ) compared to nonvaccinated challenged animals $(10 / 10$ at 14 and $28 \mathrm{dpc}$, $p<0.05)$. Vaccinated challenged animals had a significantly lower number of genomic copies of PCV2 in the nasal swab than non-vaccinated challenged animals at 14 and $28 \mathrm{dpc}(p<0.001$, Figure 1$)$. The percentage of nasal shedders was significantly lower in vaccinated challenged animals $(4 / 10$ at 14 and 28 $\mathrm{dpc})$ than in non-vaccinated challenged animals (10/10 at 14 and $28 \mathrm{dpc}, p<0.05)$. The number of genomic copies of PCV2 in the blood correlated with that of PCV2 in the nasal swabs (T01: $\mathrm{r}^{2}=0.921, p=0.042$ and T03: $\mathrm{r}^{2}=0.972$, $p=0.002)$. No PCV2 DNA was detected in the blood and nasal swabs from vaccinated non-challenged (T02) and non-vaccinated non-challenged (T04) animals throughout the experiment. No PCV1-2a DNA was detected in the blood from vaccinated (T01 and T02) and non-vaccinated (T03 and T04) animals by real-time PCR at $-14,0,14$, and $28 \mathrm{dpc}$.

\section{Anti-PCV2 lgG antibodies}

At the time of PCV2 vaccination (3 weeks of age; $-14 \mathrm{dpc}$ ), pigs in all 4 groups were seronegative against PCV2. AntiPCV2 IgG antibody titers were significantly higher in vaccinated challenged (T01) and vaccinated non-challenged (T02) animals than in non-vaccinated challenged (T03) animals at 0,14 , and $28 \mathrm{dpc}$. Anti-PCV2 IgG antibody titers were not detected in non-vaccinated non-challenged (T04) animals throughout the experiment. Anti-PCV2 IgG antibody titers did not correlate with the number of genomic copies of PCV2 in the blood (T01: $\mathrm{r}^{2}=-0.332$, $p=0.075$ and T03: $\left.\mathrm{r}^{2}=-0.105, p=0.126\right)$.

\section{Neutralizing antibodies}

At the time of PCV2 vaccination (3 weeks of age, $-14 \mathrm{dpc}$ ), no significant differences in NA titers were detected among the 4 groups. The NA titers were significantly higher in vaccinated challenged (T01) and vaccinated nonchallenged (T02) animals than in non-vaccinated challenged (T03) animals group at 0, 14, and $28 \mathrm{dpc}$. The NA titers were not detected in non-vaccinated non-challenged (T04) animals throughout the experiment (Figure 2). The NA titers correlated inversely with the number of genomic copies of PCV2 in the blood (T01: $\mathrm{r}^{2}=-0.712, p=0.012$ and T03: $\left.\mathrm{r}^{2}=-0.635, p=0.031\right)$.

\section{PCV2-specific interferon- $\boldsymbol{\gamma}$-secreting cells}

PCV2-specific IFN- $\gamma$-SCs were not observed in the isolated PBMCs of pigs from the 4 groups at $-14 \mathrm{dpc}$. PCV2-specific IFN- $\gamma$-SCs increased sharply in vaccinated challenged (T01) and vaccinated non-challenged (T02) animals at $0 \mathrm{dpc}$ ( 2 weeks after vaccination). At 0 and 14 $\mathrm{dpc}$, the mean numbers of PCV2-specific IFN- $\gamma$-SCs were significantly higher in vaccinated challenged (T01) and vaccinated non-challenged (T02) animals compared to non-vaccinated challenged (T03) animals $(p<0.05)$. At $28 \mathrm{dpc}$, the mean numbers of PCV2-specific IFN- $\gamma-$ SCs were significantly higher in vaccinated challenged (T01) than in vaccinated non-challenged (T02) and nonvaccinated challenged (T03) animals $(p<0.01)$. PCV2- 


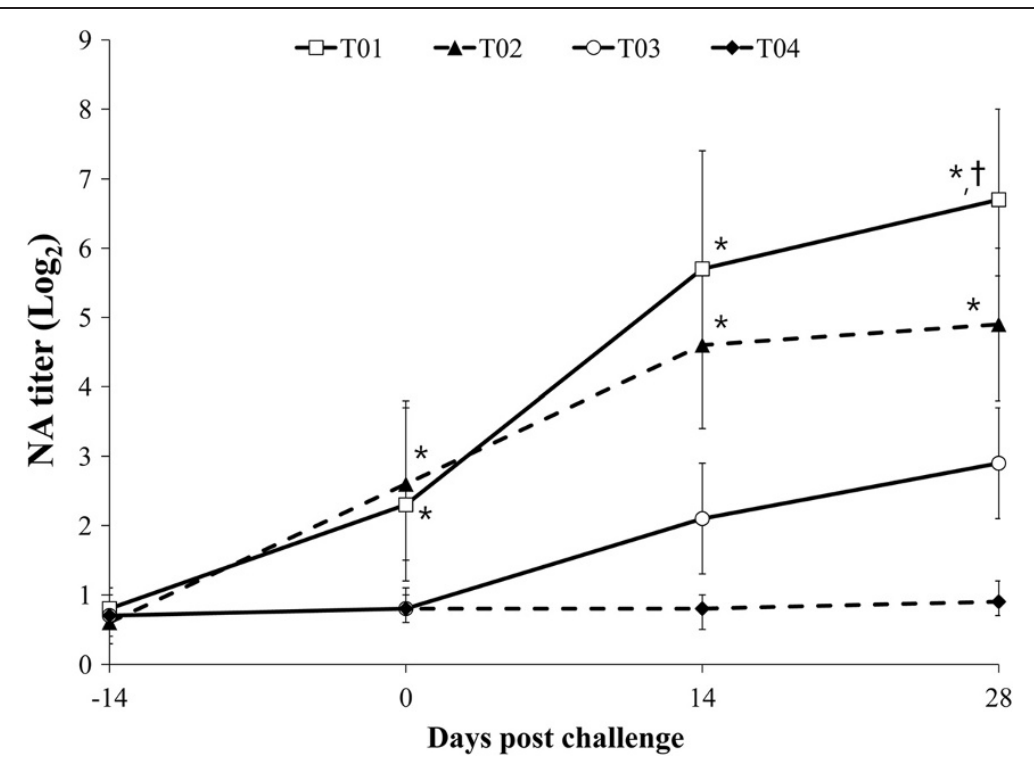

Figure 2 Mean values of the serum neutralizing antibodies (NA) titer in the different groups; vaccinated challenged (T01; $\square$ ), vaccinated non-challenged (TO2; $\Delta$ ), non-vaccinated challenged (TO3; O), and non-vaccinated non-challenged (T04; $)$ animals. Variation is expressed as the standard deviation. Significant difference (T01 and T02 vs. T03 and T04) is indicated at $p$ value $<0.01^{*}$. Significant difference (T01 vs. T02) is indicated at $p$ value $<0.05^{\dagger}$.

specific IFN- $\gamma$-SCs were not detected in the PBMCs from non-vaccinated non-challenged (T04) animals throughout the experiment (Figure 3).

PCV2-specific IFN- $\gamma$-SCs also correlated inversely with the number of genomic copies of PCV2 in the blood (T01: $\mathrm{r}^{2}=-0.685, p=0.022$ and T03: $\left.\mathrm{r}^{2}=-0.625, p=0.028\right)$.

\section{Delayed type hypersensitivity}

At $36 \mathrm{~h}$ after intradermal injection of the PCV2 antigen, vaccinated animals showed DTH responses consisted of induration and erythematous nodules (Figure 4a). Histopathologically, perivascular infiltrations of lymphocytes were observed in the dermis (Figure $4 \mathrm{~b}$ ). The specific

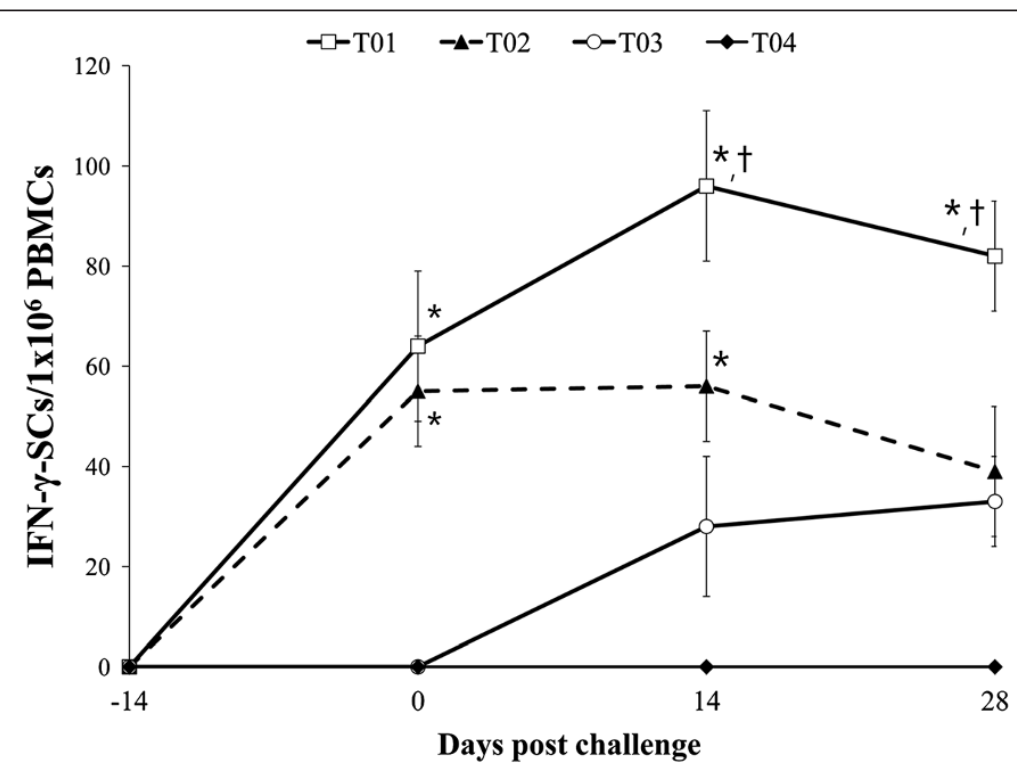

Figure 3 Mean number of porcine circovirus type 2-specific interferon- $\gamma$-secreting cells (IFN- $\gamma$-SCs) in vaccinated challenged (T01; $\square$ ), vaccinated non-challenged (T02; $\Delta$ ), non-vaccinated challenged (TO3; O), and non-vaccinated non-challenged (T04; $\$$ ) animals.

Variation is expressed as the standard deviation. Significant difference (T01 and T02 vs. T03 and T04) is indicated at $p$ value $<0.05^{*}$. Significant difference (T01 vs. T02) is indicated at $p$ value $<0.01^{\dagger}$. 

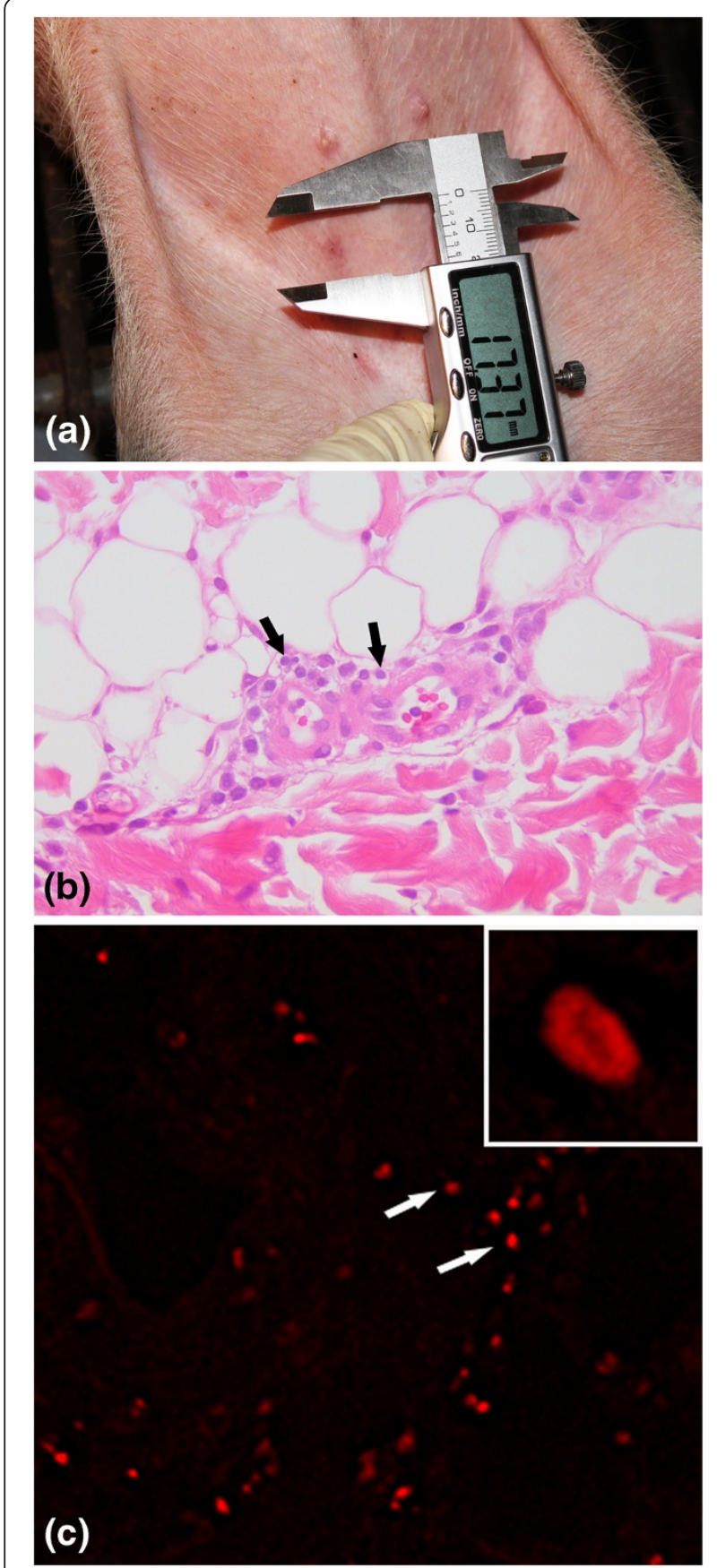

Figure 4 Delayed type hypersensitivity induced by inactivated chimeric PCV1-2 vaccine in vaccinated challenged (T01) animals. (a) erythematous lesion were grossly observed in the skin.

(b) Perivascular infiltration of lymphocytes (black arrows) was seen in the dermis. (c) $\mathrm{CD}^{+}$cells (white arrows) were seen in the perivascular zone.

fluorescence signals were observed in cells in perivascular zone (Figure 4c). The positive signals were mainly seen in the cytoplasm of cells.

The skin reaction regressed slowly at $48 \mathrm{~h}$ after injection and completely disappeared. DTH responses were not observed in the controls. The vaccinated animals in groups T01 and T02 and non-vaccinated animals in groups T03 and T04 showed DTH responses to the nonspecific mitogen PHA. The PHA DTH response size was not significantly different between vaccinated (T01 and T02) and non-vaccinated (T03 and T04) animals. The vaccinated (T01 and T02) animals displayed significantly greater PCV2-specific DTH responses than the non-vaccinated (T03 and T04) animals $(p<0.01$, Table 1). DTH responses to saline injection were not observed in any pigs. DTH response correlated with PCV2-specific IFN- $\gamma$-SCs (T01: $\mathrm{r}^{2}=0.637, p=0.015$ and T03: $\mathrm{r}^{2}=0.219, p=0.218$ ).

\section{Identification of lymphocyte subsets}

Vaccinated challenged (T01) and vaccinated nonchallenged (T02) animals showed an increase in the relative proportions of $\mathrm{CD}^{+}$and $\mathrm{CD}^{+}$cells at $0 \mathrm{dpc}$ compared with non-vaccinated challenged (T03) and non-vaccinated non-challenged (T04) animals $(p<0.05)$. Vaccinated challenged (T01) animals showed an increase in the relative proportions of $\mathrm{CD}^{+}$and $\mathrm{CD}^{+}$cells at 14 $\mathrm{dpc}$, and in the relative proportions of $\mathrm{CD}^{+}$cells at 28 dpc compared with non-vaccinated challenged (T03) and non-vaccinated non-challenged (T04) animals $(p<0.05)$. At $14 \mathrm{dpc}$, the relative proportions of $\mathrm{CD}^{+}$cells were significantly higher in vaccinated non-challenged (T02) animals than non-vaccinated challenged (T03) and nonvaccinated non-challenged (T04) animals $(p<0.05$, Figure 5). The number of $\mathrm{CD}^{+}$cells correlated with the number of PCV2-specific IFN- $\gamma$-SCs in the blood (T01: $\mathrm{r}^{2}=0.624, p=0.025$, T02: $\mathrm{r}^{2}=0.589, p=0.047$, and T03: $\left.\mathrm{r}^{2}=0.527, p=0.041\right)$. The number of $\mathrm{CD}^{+}$cells also correlated with the DTH response (T01: $\mathrm{r}^{2}=0.597$, $p=0.023$ and T03: $\left.\mathrm{r}^{2}=0.176, p=0.365\right)$.

Table 1 Mean number of delayed type hypersensitivity (DTH) response size, microscopic lymphoid lesion score and immunohistochemical Porcine circovirus type 2 (PCV2) antigen score in lymph node from vaccinated challenged (T01), vaccinated non-challenged (T02), nonvaccinated challenged (T03), and non-vaccinated nonchallenged (T04) animals

\begin{tabular}{lllll}
\hline & Groups & & \\
\cline { 2 - 5 } & T01 & T02 & T03 & T04 \\
\hline DTH & $15.38^{*} \pm 1.87$ & $14.20^{*} \pm 4.26$ & $2.19 \pm 0.08$ & $1.98 \pm 1.09$ \\
Lymphoid & $0.40^{\dagger} \pm 0.54$ & - & $1.67 \pm 0.57$ & - \\
lesion score & & & & \\
PCV2 antigen & $6.18^{\dagger} \pm 4.79$ & - & $44.12 \pm 9.07$ & - \\
score & & & & \\
\hline
\end{tabular}

${ }^{*}$ Significant difference (T01 and T02 vs. T03 and T04) is indicated at $p$ value $<0.01$. ${ }^{\dagger}$ Significant difference (T01 vs. T03) is indicated at $p$ value $<0.05$. 


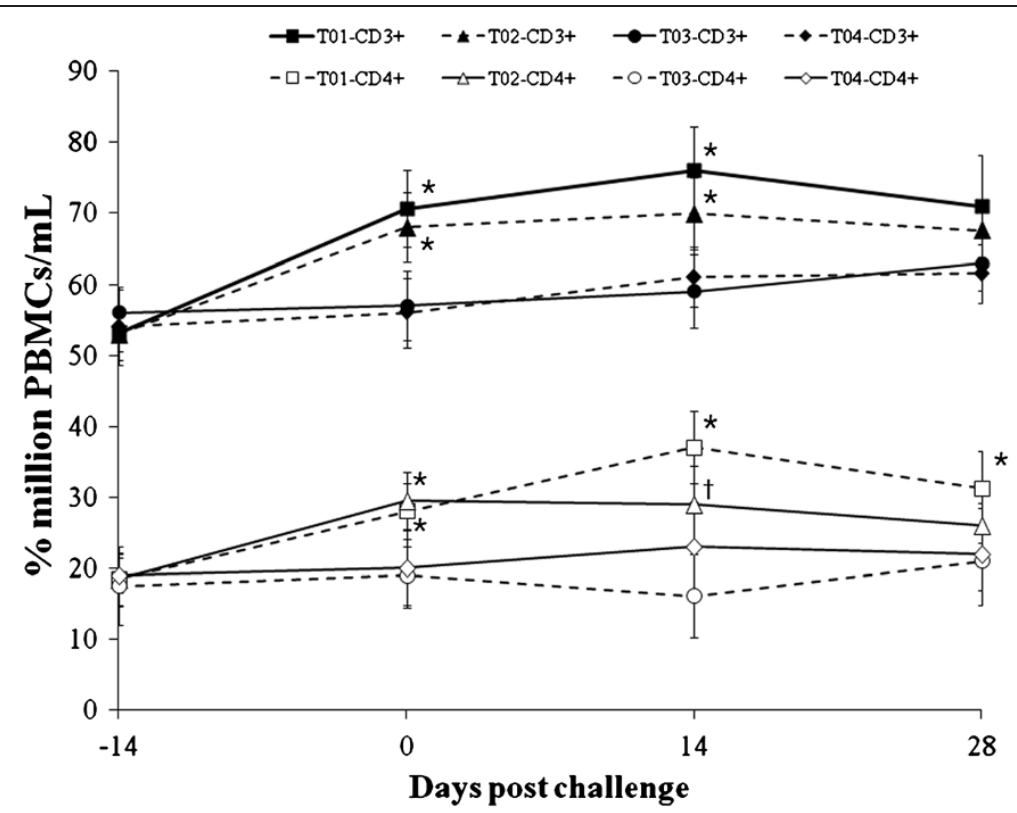

Figure 5 Lymphocyte subsets analysis in the different groups; $\mathrm{CD}^{+}(\square)$ and $\mathrm{CD}^{+}(\square)$ from vaccinated challenged (T01) animals, $\mathrm{CD} 3^{+}$ $(\Delta)$ and $\mathrm{CD}^{+}(\triangle)$ from vaccinated non-challenged $(\mathrm{TO2})$ animals, $\mathrm{CD}^{+}(\bullet)$ and $\mathrm{CD}^{+}(\mathrm{O})$ from non-vaccinated challenged $(\mathrm{T03})$ animals, and $\mathrm{CD}^{+}(\diamond)$ and $\mathrm{CD}^{+}(\diamond)$ from non-vaccinated non-challenged (T04) animals. Variation is expressed as the standard deviation. Significant difference (T01 and T02 vs. T03 and T04) is indicated at $p$ value $<0.05^{*}$. Significant difference (T02 vs. T03) is indicated at $p$ value $<0.05^{\dagger}$.

\section{Histopathology and immunohistochemistry}

The histopathological lymphoid lesion scores were significantly lower in the vaccinated challenged (T01) animals than in the non-vaccinated challenged (T03) animals $(p<0.05$, Table 1$)$. The histopathological lymphoid lesion scores correlated with the number of genomic copies of PCV2 in the blood (T01: $\mathrm{r}^{2}=0.870, p=0.041$ and T03: $\left.\mathrm{r}^{2}=0.892, p=0.023\right)$. The mean number of PCV2-positive cells per unit area of lymph node was significantly lower in vaccinated challenged (T01) animals than in non-vaccinated challenged (T03) animals $(p<0.01$, Table 1). The PCV2 antigen scores correlated with the number of genomic copies of PCV2 in the blood (T01: $\mathrm{r}^{2}=0.855, p=0.038$ and T03: $\mathrm{r}^{2}=0.872, p=0.047$ ).

\section{Discussion}

Quantitation of the PCV2 viremia could predict PCV2 infection status. Several studies have already shown that PCV2 DNA levels in serum are higher in pig with PMWS than in healthy, subclinically infected pigs [20-22]. Hence, the reduction of PCV2 viremia by the PCV2 vaccine plays a critical role in controlling PCV2 infection. In the present study, the reformulated inactivated chimeric PCV1-2 vaccine is able to induce PCV2-specific NA and IFN- $\gamma$-SCs in vaccinated animals. This protective immunity induced by the reformulated inactivated chimeric PCV1-2 vaccine correlated with the reduction of PCV2 viremia in pigs challenged experimentally with solely PCV2 as induced by other commercial PCV2 vaccines did [10,23]. However, the presence of antiPCV2 IgG antibodies did not correlate with the reduction of PCV2 viremia. The reformulated inactivated chimeric PCV1-2 vaccine also reduced the PCV2 load in nasal shedding in vaccinated animals, thereby decreasing the risk of transmission to other pigs via a nasal route and decreasing the amount of PCV2 circulating among the pigs.

IFN- $\gamma$, which is produced by PCV2-specific IFN- $\gamma$-SCs, is a key immunoregulatory cytokine that controls the differentiation of naïve $\mathrm{CD} 4^{+}$into $\mathrm{CD} 4^{+}$cells and mediates cellular immunity against viral infections [24]. Our results are further supported by the observation that elevated numbers of $\mathrm{CD} 4^{+}$cells are seen in vaccinated animals only. Lymphocyte subset analysis indicated that the numbers of $\mathrm{CD}^{+}$and $\mathrm{CD}^{+}$cells increased in vaccinated animals but the numbers of $\mathrm{CD} 4^{+}$cells decreased transiently in non-vaccinated animals. The selective loss of $\mathrm{CD}^{+}$and $\mathrm{CD} 4^{+}$cells that is observed in pigs with PMWS $[25,26]$ may impair the immune system in the pigs and result in co-infections with other viral and bacterial pathogens; co-infections are frequently observed in pigs with PMWS under field conditions [27,28]. Because $\mathrm{CD}^{+}$cells promote a DTH response [29], the DTH response observed in only vaccinated animals also supports a $\mathrm{CD} 4^{+}$cell-associated protective cellular immune response that is induced by the reformulated inactivated chimeric PCV1-2 vaccine. PCV2-specific memory $\mathrm{T}$ lymphocytes induced by this chimeric PCV1-2 vaccine 
mount DTH reactions in response to intradermal injection of the PCV2 antigen. Hence, the induction of PCV2-specific NA and IFN- $\gamma$-SCs by the reformulated inactivated chimeric PCV1-2 vaccine is the important protective immune response that leads to reduce the PCV2 viremia and control the PCV2 infection.

Well-controlled experimental studies are necessary to elucidate the protective humoral and cellular immune response induced by the reformulated inactivated chimeric PCV1-2 vaccine to reduce PCV2 viremia because the results can be affected by factors such as environment, feed, pig source, immune status and inoculums. Under field conditions, PCV2 continues to circulate among pigs within the herd and the possibility of exposure and re-exposure to the virus by horizontal transmission occurs once one animal becomes infected. To our knowledge this is the first demonstration of protective humoral and cellular immunity induced by the reformulated inactivated chimeric PCV1-2 vaccine and its effect on reduction of PCV2 viremia by vaccination.

\section{Conclusions}

The reformulated inactivated chimeric PCV1-2 vaccine is able to induce PCV2-specific NA and IFN- $\gamma$-SCs in vaccinated animals. This protective immunity induced by the reformulated inactivated chimeric PCV1-2 vaccine correlated with the reduction of PCV2 viremia in pigs challenged experimentally with solely PCV2.

\section{Competing interests}

The authors state that there are no competing interests related to the present study.

\section{Authors' contributions}

HWS performance of the experimental trials, data analysis and writing of the manuscript, YO, KH and CP preparation of the inoculum and lab analysis, CC development of protocol, design of the study, review of the final manuscript, approval for publication. All authors read and approved the final manuscript.

\section{Acknowledgements}

This research was supported by the Pfizer Animal Health Inc. This research was also supported by contract research funds of the Research Institute for Veterinary Science (RIVS) from the College of Veterinary Medicine and by Brain Korea 21 Program for Veterinary Science in the Republic of Korea. Authors thank Pfizer Animal Health Korea personnel; Dr. Sung-Kyung You, Dr. Gwang-Eun Lee, and Dr. Su-Jin Park.

Received: 2 July 2012 Accepted: 17 October 2012

Published: 19 October 2012

\section{References}

1. Chae C: Commercial porcine circovirus type 2 vaccines: Efficacy and clinical application. Vet J, doi:10.1016/j.tvjl.2012.06.031. in press.

2. Chae C: A review of porcine circovirus 2-associated syndromes and diseases. Vet J 2005, 169:326-336.

3. Chae C: Postweaning multisystemic wasting syndrome: a review of aetiology, diagnosis and pathology. Vet J 2004, 168:41-49.

4. Fenaux M, Opriessnig T, Halbur PG, Elvinger F, Meng XJ: A chimeric porcine circovirus (PCV) with the immunogenic capsid gene of the pathogenic PCV type 2 (PCV2) cloned into the genomic backbone of the nonpathogenic PCV1 induces protective immunity against PCV2 infection in pigs. J Virol 2004, 78:6297-6303.
5. Gagnon CA, Music N, Fontaine G, Tremblay D, Harel J: Emergence of a new type of porcine circovirus in swine (PCV): A type 1 and type 2 PCV recombinant. Vet Microbiol 2010, 144:18-23.

6. Meerts P, van Gucht S, Cox E, Vandebosch A, Nauwynck HJ: Correlation between type of adaptive immune response against porcine circovirus type 2 and level of virus replication. Viral Immunol 2005, 18:333-341.

7. Meerts P, Misinzo G, Lefebvre D, Nielsen J, Botner A, Kristensen CS, Nauwynck H: Correlation between the presence of neutralizing antibodies against porcine circovirus 2 (PCV2) and protection against replication of the virus and development of PCV2-associated disease. BMC Vet Res 2006, 2:6-16.

8. Fort M, Olvera A, Sibila M, Segalés J, Mateu E: Detection of neutralizing antibodies in postweaning multisystemic wasting syndrome (PMWS)affected and non-PMWS-affected pigs. Vet Microbiol 2007, 125:244-255

9. Fort M, Fernandes LT, Nofrarias M, Diaz I, Sibila M, Pujols J, Mateu E, Segalés J: Development of cell-mediated immunity to porcine circovirus type 2 (PCV2) in caesarean-derived, colostrum-deprived piglets. Vet Immunol Immunopathol 2009, 129:101-107.

10. Fort $M$, Sibila $M$, Perez-Martin E, Nofrarias M, Mateu E, Segalés J: One dose of a porcine circovirus 2 (PCV2) sub-unit vaccine administered to 3week-old conventional piglets elicits cell-mediated immunity and significantly reduces PCV2 viremia in an experimental model. Vaccine 2009, 27:4031-4037.

11. Oh Y, Seo HW, Han K, Park C, Chae C: Protective effect of the maternally derived porcine circovirus type 2(PCV2)-specific cellular immune response in piglets by dam vaccination against PCV2 challenge. $J$ Gen Virol 2012, 93:1556-1562.

12. Opriessnig T, Patterson AR, Madson DM, Pal N, Halbur PG: Comparison of efficacy of commercial one dose and two dose PCV2 vaccines using a mixed PRRSV-PCV2-SIV clinical infection model 2-3-months post vaccination. Vaccine 2009, 27:1002-1007.

13. Shen HG, Beach NM, Huang YW, Halbur PG, Meng XJ, Opriessnig T: Comparison of commercial and experimental porcine circovirus type 2 (PCV2) vaccines using a triple challenge with PCV2, porcine reproductive and respiratory syndrome virus (PRRSV), and porcine parvovirus (PPV). Vaccine 2010, 43:5960-5966.

14. Gagnon CA, del Castillo JR, Music N, Fontaine G, Harel J, Tremblay D: Development and use of a multiplex real-time quantitative polymerase chain reaction assay for detection and differentiation of porcine circovirus-2 genotypes $2 \mathrm{a}$ and $2 \mathrm{~b}$ in an epidemiological survey. J Vet Diagn Invest 2008, 20:545-558.

15. Kim D, Kim CH, Han K, Seo HW, Oh Y, Park C, Kang I, Chae C: Comparative efficacy of commercial mycoplasma hyopneumoniae and porcine circovirus 2 (PCV2) vaccines in pigs experimentally infected with $M$. Hyopneumoniae and PCV2. Vaccine 2011, 29:3206-3212.

16. Pogranichnyy RM, Yoon KJ, Harms PA, Swenson SL, Zimmerman JJ, Sorden SD: Characterization of immune response of young pigs to porcine circovirus type 2 infection. Viral Immunol 2000, 13:143-153.

17. Rodriguez-Arrioja GM, Segalés J, Balasch M, Rosell C, Quintant J, Folch JM, Plana-Duran J, Mankertz A, Domingo M: Serum antibodies to porcine circovirus type 1 and type 2 in pigs with and without PMWS. Vet Rec 2000, 146:762-764.

18. Williams PP: Immunomodulating effects of intestinal absorbed maternal colostral leukocytes by neonatal pigs. Can Vet Res 1993, 57:1-8.

19. Sosa GA, Quiroga MF, Roux ME: Flow cytometric analysis of Tlymphocytes from nasopharynx-associated lymphoid tissue (NALT) in a model of secondary immunodeficiency in wistar rats. Immunobiology 2009, 214:384-391.

20. Ladekjær-Mikkelsen AS, Nielsen J, Stadejek T, Storgaad T, Krakowka S, Ellis J, McNeilly F, Allan G, Botner A: Reproduction of postweaning multisystemic wasting syndrome (PMWS) in immunostimulated and nonimmunostimulated 3-week-old piglets experimentally infected with porcine circovirus type 2 (PCV2). Vet Microbiol 2002, 89:97-114.

21. Liu Q, Wang L, Willson P, Babiuk A: Quantitative, competitive PCR analysis of porcine circovirus DNA in serum from pigs with postweaning multisystemic wasting syndrome. J Clin Microbiol 2000, 38:3474-3477.

22. Rosell C, Segalés J, Plana-Duran J, Balasch M, Rodriguez-Arrioja GM, Kennedy S, Allan GM, McNeilly F, Latimer KS, Domingo M: Pathological, immunohistochemical, and in-situ hybridization studies of natural cases of postweaning multisystemic wasting syndrome (PMWS) in pigs. $J$ Comp Pathol 1999, 120:59-78. 
23. Opriessnig T, Patterson AR, Madson DM, Pal N, Ramamoorthy S, Meng XJ,

Halbur PG: Comparison of the effectiveness of passive (dam) versus active (piglet) immunization against porcine circovirus type 2 (PCV2) and impact of passively derived PCV2 vaccine-induced immunity on vaccination. Vet Microbiol 2010, 142:177-183.

24. Schroder K, Hertzog PJ, Ravasi T, Hume DA: Interferon- - : an overview of signals, mechanisms and functions. J Leuk Biol 2004, 75:163-189.

25. Nielsen J, Vincent IE, Bøtner A, Ladekaer-Mikkelsen AS, Allan G, Summerfield A, Mc-Cullough KC: Association of lymphopenia with porcine circovirus type 2 induced postweaning multisystemic wasting syndrome (PMWS). Vet Immunol Immunopathol 2003, 92:97-111.

26. Segalés J, Alonso F, Rosell C, Pastor J, Chianini F, Campos E, Lopez-Fuertes L, Quintana J, Rodriquez-Arrioja G, Calsamiglia M, Pujols J, Domingues J, Domingo M: Changes in peripheral blood leukocyte populations in pigs with natural postweaning multisystemic wasting syndrome (PMWS). Vet Immunol Immunopathol 2001, 81:37-44.

27. Kim J, Chung HK, Jung T, Cho WS, Choi C, Chae C: Postweaning multisystemic wasting syndrome of pigs in Korea: prevalence, microscopic lesions and coexisting microorganisms. J Vet Med Sci 2002, 64:57-62.

28. Pallares FJ, Halbur PG, Opriessnig T, Sorden SD, Villar D, Janke BH, Yaeger MJ, Larson DJ, Schwartz KJ, Yoon KJ, Hoffman LJ: Porcine circovirus type 2 (PCV-2) coinfections in US field cases of postweaning multisystemic wasting syndrome (PMWS). J Vet Diagn Invest 2002, 14:515-519.

29. Sanders VM: Epigenetic regulation of Th1 and Th2 cell development. Brain Behav Immun 2006, 20:317-324.

doi:10.1186/1746-6148-8-194

Cite this article as: Seo et al:: Reduction of porcine circovirus type 2 (PCV2) viremia by a reformulated inactivated chimeric PCV1-2 vaccineinduced humoral and cellular immunity after experimental PCV2 challenge. BMC Veterinary Research 2012 8:194.

\section{Submit your next manuscript to BioMed Central and take full advantage of:}

- Convenient online submission

- Thorough peer review

- No space constraints or color figure charges

- Immediate publication on acceptance

- Inclusion in PubMed, CAS, Scopus and Google Scholar

- Research which is freely available for redistribution 